1 Division of Biosciences, University College London, London, UK

2 Royal Free London NHS Foundation Trust, London, UK

3 Epidemic Diseases Research Group Oxford, University of Oxford, Oxford, UK

4 Department of Health Services Research and Policy, London School of Hygiene and Tropical Medicine, London, UK

5 NIHR Health Protection Research Unit in Emerging and Zoonotic Infections and Institute of Infection, Veterinary and Ecological Sciences, University of Liverpool, Liverpool, UK

6 Respiratory Medicine, Alder Hey Children's Hospital, Liverpool, UK

7 Institute of Population Health, University of Liverpool, Liverpool, UK

Correspondence to: A Crozier alexander.crozier.20@ucl.ac.uk Cite this as: BMJ 2021;374:n1625 http://dx.doi.org/10.1136/bmj.n1625 Published: 01 July 2021

\title{
Could expanding the covid-19 case definition improve the UK's pandemic response?
}

Alex Crozier and colleagues evaluate the potential opportunities and challenges of expanding the
symptom list linked to self-isolation and testing as vaccines are rolled out Alex Crozier, ${ }^{1}$ Jake Dunning, ${ }^{2,3}$ Selina Rajan, ${ }^{4}$ Malcolm G Semple, ${ }^{5,6}$ lain E Buchan ${ }^{5,7}$

During the covid-19 pandemic the British public has been instructed: "If you have a high fever, a new continuous cough, or you've lost your sense of smell or taste or its changed, self-isolate and get a test." ${ }^{1}$ Yet these symptoms are just a few of many described by those infected with SARS-CoV-2. ${ }^{2-5}$ Many people with mild-to-moderate disease don't have these symptoms (initially), and other symptoms often manifest earlier. ${ }^{36}$ Most spread is from symptomatic cases around the time of symptom onset, ${ }^{7-11}$ and interrupting transmission depends on early identification and isolation of contagious individuals. ${ }^{12} 13$ The narrow UK case definition therefore limits this detection, restricting the effectiveness of the test, trace, and isolate programme. ${ }^{814} 15$

As vaccination progresses and social mixing increases, infections are now highest among young, unvaccinated, or partially vaccinated people, who are also more likely to experience 'unofficial' symptoms. ${ }^{16} 17$ Variants are adding further to transmission, as predicted, with potential for another wave of hospital admissions and deaths. ${ }^{18}$ Improvements in transmission control are urgently needed. Here, we build on calls to broaden the UK's covid-19 case definition, ${ }^{519}$ analysing the potential to improve self-isolation and symptomatic testing guided by a case definition fit for the vaccination era.

\section{Updating the UK's clinical case definition}

The European Centre for Disease Prevention and Control described a breadth of symptoms associated with mild-to-moderate covid-19, the most commonly reported being headache $(70 \%)$, nasal obstruction $(68 \%)$, weakness or fatigue $(63 \%)$, myalgia $(63 \%)$, rhinorrhoea (60\%), gustatory dysfunction (54\%), and sore throat (53\%). ${ }^{20}$ Many infected people do not present with the symptoms used in the UK case definition: loss of taste or smell, a cough, or fever which, before vaccination rollout were reported by $70 \%, 63 \%$, and $45 \%$ of symptomatic cases, respectively. ${ }^{321}$ While restricting access to symptomatic testing to those with "official" symptoms may control the volume of testing, this narrow definition is now likely to impede control of transmission.

Critically, unofficial symptoms often manifest earlier. ${ }^{9}$ In a recent population based study in Arizona, US, the most commonly reported first symptoms were sore throat (19\%), headache (16\%), cough (13\%), runny nose or cold-like symptoms (12\%), and fatigue $(12 \%){ }^{22}$ These symptoms are more common in school age children ${ }^{16}$ and younger people, ${ }^{17}$ who now account for an even greater proportion of transmission because older people are vaccinated.

The World Health Organization ${ }^{2}$ and Centers for Disease Control and Prevention ${ }^{4}$ already include nine and 11 more case defining symptoms, respectively, than the UK. Greater testing capacity is now available to accommodate a wider case definition in the UK, particularly with rapid antigen tests. However, rapid tests are officially being used only for self-testing (at home or at testing centres) by people without symptoms, ${ }^{23} 24$ although some people with wider symptoms may also be using them. ${ }^{25}$ Symptomatic testing using reverse transcription polymerase chain reaction (RT-PCR) tests meanwhile is open only to those declaring a high temperature, a new continuous cough, or a loss or change in sense of smell, and to confirmed contacts of RT-PCR positive cases.

The UK's narrow clinical case definition impedes not only the identification of cases but also the understanding of SARS-CoV-2 transmission. Although infected individuals without symptoms can clearly pass on the virus, ${ }^{26}$ the characterisation of asymptomatic infection and transmission has been poor. ${ }^{3}$ It is important to distinguish between those not experiencing symptoms throughout infection (persistently asymptomatic), becoming infectious before symptoms manifest (presymptomatic), or having only unofficial or subtle symptoms (pauci-symptomatic). Persistently asymptomatic cases probably account for less than $20 \%$ of infections, and these people may be 3-25 times less likely than those with symptoms to pass on the virus. 7 $^{-11}$

Real world evidence suggests presymptomatic and (official and unofficial) symptomatic cases drive transmission more than asymptomatic cases. ${ }^{7-11}$ It seems counterintuitive, therefore, to have no official UK guidance on wider covid-19 symptoms, or to offer different testing routes for those with official symptoms and those with no symptoms, with nothing in- between. People with unofficial symptoms can bypass the rules to get a test-legitimising this choice could be helpful.

Concerns have been raised over testing capacity, false negative rapid test results, and non-compliance with self-isolation. ${ }^{2324}$ However, the benefits of identifying more cases sooner are likely to be substantial. The Scientific Advisory Group for Emergencies (SAGE) recommended "prioritising rapid testing of symptomatic people is likely to have a greater impact on identifying positive cases and reducing 
transmission than frequent testing of asymptomatic people in an outbreak area." 27

Testing people with a single, non-specific symptom could, of course, overwhelm or waste capacity. Indeed, September 2020 government advisory groups ${ }^{28}{ }^{29}$ considered data from the First Few Hundred Study ${ }^{30}$ and Covid Symptom Study App to reason against expanding eligibility for symptomatic testing. The data suggested expanding the definition would decrease symptom specificity from $97 \%$ to $94 \%$ while only marginally increasing symptom sensitivity from $85 \%$ to $95 \%$. However, more recent evidence on symptom combinations warrants reconsidering the case definition, especially since vaccination means the population most likely to be infected and transmit will now be younger or partially immunised, and so less likely to experience severe disease or official symptoms.

Combinations of symptoms could be used to help identify more cases sooner without overwhelming testing capacity. An age stratified approach derived from the React study selected chills (all ages), headache (5-17 years), appetite loss ( $\geq 18$ years), and muscle aches (18-54 years) as jointly predictive of positive RT-PCR results, together with the official symptoms. ${ }^{5}$ The authors concluded that triage based on these symptoms would identify more cases than the current approach, at any level of testing.

The Virus Watch cohort suggested that using a wider symptom definition captured cases a day earlier than the current definition, on average, ${ }^{31}$ a critical time difference for preventing transmission. The Covid Symptom Study App was used to identify optimal symptom combinations for capturing most cases with fewest tests, and found that within three days of symptom onset, dyspnoea plus the official symptom combination (cough, fever, loss of smell or taste) identified only $69 \%$ of symptomatic cases and required 47 tests for each case identified. ${ }^{22}$ The combination with the highest coverage (fatigue, loss of smell or taste, cough, diarrhoea, headache, sore throat) identified $96 \%$ of symptomatic cases (requiring 96 tests per case identified). ${ }^{32}$ This combination of symptoms would increase the number of cases captured by symptomatic testing by over a third, and would likely result in earlier identification of many cases, ${ }^{22}$ potentially containing transmission more as we reopen society.

\section{Implementing an updated clinical case definition}

Expanding the case definition is likely to increase demand for testing and numbers self-isolating. The system-wide effects would be complex, requiring careful implementation. ${ }^{33}$ Any change must neither overwhelm NHS Test and Trace nor impede existing symptomatic testing. Instructions such as "isolate if you have case defining symptoms, regardless of test status” must not lose clarity despite more complex lists of symptoms. Potential harms from false negative or positive results need mitigation. While it is essential to consider the pre-test probability of infection (based on background prevalence, epidemiological history, and clinical presentation) and the performance of the test used, ${ }^{3435}$ a substantial net reduction in transmission is likely if more symptomatic people are identified and isolate sooner.

The UK's decision to adopt a narrow case definition was based on ease of communication, avoiding confusion with other infections, and preserving testing capacity. This situation is now

different-testing capacity is high. The emergence of the delta

variant and the potential evolution of more transmissible or vaccine resistant variants means that, even with vaccination, further waves of cases, hospital admissions, and deaths may ensue. ${ }^{18}$ Mitigating these waves, and the potential for enduring transmission, ${ }^{36}$ requires agile intervention to minimise the risks of vaccine escape variants, long covid, further NHS disruption, and harms from restrictions. To realise the benefits of a wider case definition it will be necessary to revise policies for testing and self-isolation.

Since RT-PCR capacity is limited, and quick turnaround is vital, we suggest dynamic targeting of RT-PCR testing, guided by continuous review of symptoms, transmission patterns, variants, vaccination uptake, and circulation of other respiratory viruses. Routinely collected data could be used to adapt testing eligibility, access, and communications systematically and quickly. . $^{-39}$ Communication is particularly important considering that only half the public can correctly identify the existing official covid-19 symptoms..$^{40}$ Data intensive, intelligence-led adaptation of the test, trace, and isolate system could make an important contribution to the UK's pandemic responses while we wait for the vaccination programme to progress as far as possible and for covid-19 to abate.

\section{Refining test, trace, and isolate}

Given the heterogeneity in SARS-CoV-2 transmission, ${ }^{81415}$ whereby fewer than $20 \%$ of cases may account for more than $80 \%$ of transmission, reopening society ahead of maximum vaccination coverage requires better identification and self-isolation of infectious cases to contain emerging clusters. To achieve this, the NHS Test and Trace system must increase the proportion of cases tested (and isolated) early in their infection and trace more contacts before onwards transmission. Early, active case finding combined with enhanced contact tracing (including backwards to identify source of infection), ${ }^{14}$ effective symptom monitoring, ${ }^{41}$ and prompt contact testing ${ }^{42}$ can also reduce transmission..$^{13}$ Repeat testing of contacts may usefully replace isolation for those without symptoms. 4344 Viral sequencing can also help trace clusters back to their source, 45 as well as targeting resources to identify and contain more transmissible or vaccine resistant variants. Hyper-local approaches-involving communities at neighbourhood or street level, in faith groups, and other local contexts-are also vital.

Testing uptake among people with symptoms has been low, and engagement with testing and isolation has been lowest in communities with the highest prevalence of SARS-CoV-2 and the gravest consequences from covid-19. ${ }^{2324} 40$ Effective support, including prompt financial help, during self-isolation is the key to controlling transmission. ${ }^{4647}$ To make the most of an expanded case definition, public health and NHS systems must integrate more at both local and national levels, ${ }^{48-50}$ enabling nimbler, more equitable targeting of test-trace-isolate resources ${ }^{51}{ }^{52}$ and surge vaccination. ${ }^{53}$ In addition, combinations of RT-PCR and rapid antigen tests may be helpful in reducing delays between symptom onset, testing, self-isolation, and initiation of contact tracing. ${ }^{39}$

Vaccinations alone are unlikely to end the pandemic. New, more transmissible and (partially) vaccine resistant variants may spread through susceptible populations causing high hospital admission rates. Inequities in vaccination are also shifting the burden of disease and disruption to the most disadvantaged communities, who are also harmed most by covid-19 restrictions. To reopen society with greater speed and fairness, control of transmission must improve. This starts with an expanded and more context appropriate case definition and rests on adaptive, locally grounded, and information-led public health responses.

\section{Key messages}

- Covid-19 is associated with a wide range of symptoms

- Many patients do not experience the UK's official case defining symptoms, initially, or ever, and other symptoms often manifest earlier 
- Limiting the symptomatic testing to those with these official symptoms will miss or delay identification of many covid-19 cases, hampering efforts to interrupt transmission

- Expanding the clinical case definition of covid-19, the criteria for self-isolation, and eligibility for symptomatic testing could improve the UK's pandemic response

- Dynamic targeting based on data could avoid overloading resources

Contributors and sources: The authors have broad experience and direct involvement in covid-19 responses. AC has expertise in developing and troubleshooting diagnostic assays and improved covid-19 testing programmes for sports organisations. JD was a national incident director for covid-19 at Public Health England. SR has supported the Public Health England regional response, including managing outbreaks in care homes and educational institutions. MGS is a member of the New and Emerging Respiratory Virus Threats Advisory Group (NERVTAG) and regularly attends SAGE covid-19. $\mathrm{IB}$ is a NIHR senior investigator and led the evaluation of the Liverpool community testing pilot. AC conceived the article and wrote the first draft in discussion with all authors, and with input from Martin McKee. All authors edited drafts. IB acts as guarantor.

Competing interests: We have read and understood BMJ policy on declaration of interests and have the following interests to declare: IEB and MGS received grant funding from the UK Department of Health and Social Care to evaluate lateral flow tests in the Liverpool pilot. IEB reports fees from AstraZeneca as chief data scientist adviser via Liverpool University outside the submitted work. MGS is chair of the infectious disease scientific advisory board and a minority shareholder in Integrum Scientific which has interests in covid-19 testing, and reports grants from the NIHR, the Medical Research Council, and the Health Protection Research Unit in Emerging and Zoonotic Infections, University of Liverpool.

Provenance and peer review: Not commissioned; externally peer reviewed.

Get a free PCR test to check if you have coronavirus. https://www.gov.uk/get-coronavirus-test

2 WHO. WHO covid-19: case definitions. Dec 2020. https:/www.who.int/publications/i/item/WHO2019-nCoV-Surveillance_Case_Definition-2020.2

3 Meyerowitz EA, Richterman A, Bogoch II, Low N, Cevik M. Towards an accurate and systematic characterisation of persistently asymptomatic infection with SARS-CoV-2. Lancet Infect Dis 2021;21:e163-9.doi: 10.1016/S1473-3099(20)30837-9. pmid: 33301725

4 Centers for Disease Control and Prevention. Coronavirus disease 2019 (covid-19) 2020 interim case definition. 2020. https://wwwn.cdc.gov/nndss/conditions/coronavirus-disease-2019-covid19/case-definition/2020/08/05/

5 Elliott J, Whitaker M, Bodinier B, et al. Symptom reporting in over 1 million people: community detection of COVID-19. 2021. https://spiral.imperial.ac.uk/bit-

stream/10044/1/85969/5/COVID_19_symptoms_REACT_1_V2.pdf

6 Sudre CH, Lee KA, Loachlainn MN, etal. Symptom clusters in covid-19: a potential clinical prediction tool from the COVID Symptom study app.medRxiv 2020. [Preprint.] doi: 10.1101/2020.06.12.20129056

7 Qiu X, Nergiz Al, Maraolo AE, etal. Defining the role of asymptomatic and pre-symptomatic SARS-CoV-2 transmission-a living systematic review. Clin Microbiol Infect 2021;27:511-9. doi: 10.1016/j.cmi.2021.01.011. pmid: 33484843

8 Koh WC, Naing L, Chaw L, etal. What do we know about SARS-CoV-2 transmission? A systematic review and meta-analysis of the secondary attack rate and associated risk factors. PLoS One 2020;15:e0240205. doi: 10.1371/journal.pone.0240205. pmid: 33031427

9 Madewell Z), Yang Y, Longini IM, JrHalloran ME, Dean NE. Household transmission of SARS-CoV-2: a systematic review and meta-analysis. JAMA Netw Open 2020;3:e2031756. doi: 10.1001/jamanetworkopen.2020.31756. pmid: 33315116

10 Cevik M, Kuppalli K, Kindrachuk J, Peiris M. Virology, transmission, and pathogenesis of SARS-CoV-2. BMJ 2020;371:m3862. doi: 10.1136/bmj.m3862. pmid: 33097561

11 Buitrago-Garcia D, Egli-Gany D, Counotte MJ, etal. Occurrence and transmission potential of asymptomatic and presymptomatic SARS-CoV-2 infections: a living systematic review and meta-analysis. PLoS Med 2020;17:e1003346. doi: 10.1371/journal.pmed.1003346. pmid: 32960881

12 Kucharski AJ, Klepac P, Conlan AJK, etalCMMID COVID-19 working group. Effectiveness of isolation, testing, contact tracing, and physical distancing on reducing transmission of SARS-CoV-2 in different settings: a mathematical modelling study. Lancet Infect Dis 2020;20:1151-60. doi: 10.1016/S1473-3099(20)30457-6 pmid: 32559451

13 Crozier A, Rajan S, Buchan I, McKee M. Put to the test: use of rapid testing technologies for covid-19. BMJ2021;372:n208. doi: 10.1136/bmj.n208. pmid: 33536228

14 Endo A, Leclerc QJ, Knight GM, etalCentre for the Mathematical Modelling of Infectious Diseases COVID-19 Working Group. Implication of backward contact tracing in the presence of overdispersed transmission in COVID-19 outbreaks. Wellcome Open Res 2021:5:239. doi: 10.12688/wellcomeopenres.16344.3 pmid: 33154980

15 Taube JC, Miller PB, Drake JM. An open-access database of infectious disease transmission trees to explore superspreader epidemiology.medRxiv2021:2021.01.11.21249622. [Preprint.] https://doi.org/10.1101/2021.01.11.21249622
16 Parcha V, Booker KS, Kalra R, etal. A retrospective cohort study of 12,306 pediatric COVID-19 patients in the United States. Sci Rep 2021;11:10231. doi: 10.1038/s41598-021-89553-1 pmid: 33986390

17 Swann OV, Holden KA, Turtle L, etallSARIC4C Investigators. Clinical characteristics of children and young people admitted to hospital with covid-19 in United Kingdom: prospective multicentre observational cohort study. BMJ 2020;370:m3249. doi: 10.1136/bmj.m3249 pmid: 32960186

18 Scientific Pandemic Influenza Group on Modelling, Operational Subgroup. SPI-M-O: Summary of further modelling of easing restrictions - Roadmap Step 2, 31 March 2021.

https://www.gov.uk/government/publications/spi-m-o-summary-of-further-modelling-of-easingrestrictions-roadmap-step-2-31-march-2021

19 Sohal A. Open letter to Chris Whitty and Susan Hopkins: change covid-19 case definition in line with WHO to save lives. BMJ2021;372:n283. doi: 10.1136/bmi.n283. pmid: 33514502

20 European Centre for Disease Prevention and Control. Clinical characteristics of covid-19. https://www.ecdc.europa.eu/en/covid-19/latest-evidence/clinical (2020)

21 Wells PM, Doores KJ, Couvreur S, etal. Estimates of the rate of infection and asymptomatic COVID-19 disease in a population sample from SE England. J Infect 2020;81:931-6. doi: 10.1016/j.jinf.2020.10.011 pmid: 33068628

22 Khan SM, Farland LV, Austhof E, etal. Symptoms of COVID-19 in a population-based cohort study.medRxiv 2021 [Preprint.]. doi: https://doi.org/10.1101/2021.03.20.21254040

23 Raffle A, Taylor-Phillips S, Stitch A. Mapping the outcomes of covid-19 testing reveals the best opportunities for system improvement. BMJ Opinion 9 Apr 2021.

https://blogs.bmj.com/bmj/2021/04/09/mapping-the-outcomes-of-covid-19-testing-programmesreveals-the-best-opportunities-for-improvement/

24 Department of Health and Social Care. Liverpool covid-19 community testing pilot-interim evaluation report. 2020. https://www.gov.uk/government/publications/liverpool-covid-19-community-testing-pilot-interim-evaluation-report-summary

25 Dagli M. Inappropriate use of lateral flow tests by symptomatic patients. Electronic response to: Covid-19: MHRA is concerned over use of rapid lateral flow devices for mass testing (BM) 2021;373:n1090). https://www.bmj.com/content/373/bmj.n1090/rr

26 Johansson MA, Quandelacy TM, Kada S, etal. SARS-CoV-2 transmission from people without covid-19 symptoms. JAMA Netw Open 2021;4:e2035057. doi: 10.1001/jamanetworkopen.2020.35057 pmid: 33410879

27 SAGE. 56th meeting on covid-19, 10 Sep 2020. https://assets.publishing.service.gov.uk/government/uploads/system/uploads/attachment_data/file/928699/S0740_Fifty-sixth_SAGE_meeting_on_Covid-19.pdf

28 NERVTAG: community case definitions for COVID-19, 2 September 2020 https:/hww.gov.uk/government/publications/nervtag-community-casedefinitions-for-covid-19-2-september-2020

29 SAGE 57 minutes: Coronavirus (COVID-19) response, 17 September 2020. https://www.gov.uk/government/publications/sage-57-record-of-meeting-on-covid-19-17september-2020

30 Boddington NL, etal. COVID-19 in Great Britain: epidemiological and clinical characteristics of the first few hundred (FF100) cases: a descriptive case series and case control analysis.medRxiv 2020 [Preprint.] doi: 10.1101/2020.05.18.20086157

31 Fragaszy E, etal. Symptom profiles and accuracy of clinical definitions for COVID-19 in the community. Results of the Virus Watch community cohort.medRxiv2021:2021.05.14.21257229 [Preprint.] https://www.medrxiv.org/content/10.1101/2021.05.14.21257229v2

32 Antonelli M, Capdevila J, Chaudhari A, etal. Optimal symptom combinations to aid COVID-19 case identification: analysis from a community-based, prospective, observational cohort. J Infect 2021;82:384-90. doi: 10.1016/j.jinf.2021.02.015 pmid: 33592254

33 Tulloch J, Micocci M, Buckle P, etal. Enhanced lateral flow testing strategies in care homes are associated with poor adherence and were insufficient to prevent covid-19 outbreaks: results from a mixed methods implementation study.SSRN2021 [Preprint.] doi: 10.2139/ssrn.3822257

34 Watson J, Whiting PF, Brush JE. Interpreting a covid-19 test result. BMJ 2020;369:m1808. doi: 10.1136/bmj.m1808. pmid: 32398230

35 Peeling RW, Olliaro PL, Boeras DI, Fongwen N. Scaling up COVID-19 rapid antigen tests: promises and challenges. Lancet Infect Dis 2021;3099:21-6.pmid: 33636148

36 De Gruchy J. We need the tools to address Covid enduring transmission. Local Government Chronicle, 2021.

37 Chiu I-M, Cheng C-Y, Zhang H, Lin CR. Self-screening to reduce medical resource consumption facing the COVID-19 pandemic. Emerg Med /2020;37:255. doi: 10.1136/emermed-2020-209647 pmid: 32273299

38 HSE COVID-19 Antigen Testing Working Group. Covid-19 antigen test validation summary report. 2021. https://www.hse.ie/eng/services/publications/healthprotection/hse-covid-19-antigen-testvalidation-report-june-2021.pdf

39 Berger A, Nsoga MTN, Perez-Rodriguez FJ, etal. Diagnostic accuracy of two commercial SARS-CoV-2 antigen-detecting rapid tests at the point of care in community-based testing centers. medRxiv2020. [Preprint.] doi: 10.1101/2020.11.20.20235341

40 Smith LE, Potts HWW, Amlôt R, Fear NT, Michie S, Rubin GJ. Adherence to the test, trace, and isolate system in the UK: results from 37 nationally representative surveys. BMJ2021;372:n608. doi: 10.1136/bmj.n608 pmid: 33789843

41 Perrault A, Charpignon M, Gruber J, Tambe M, Majumder MS. Designing efficient contact tracing through risk-based quarantining.medRxiv 2020. [Preprint.] doi: 10.1101/2020.11.16.20227389 
42 Centers for Disease Control and Prevention. Options to reduce quarantine for contacts of personswith SARS-CoV-2 infection using symptom monitoring and diagnostic testing. https://www.cdc.gov/coronavirus/2019-ncov/science/science-briefs/scientific-brief-options-toreduce-quarantine.html

43 SPI-M-O. Statement on daily contact testing. 3 Mar 2021 https://assets.publishing.service.gov.uk/government/uploads/system/uploads/attachment_data/file/976324/S1146_SPI-MO_Daily_contact_testing.pdf

44 Love N, Reddy D, Turner C, etal. The acceptability of testing contacts of confirmed COVID-19 cases using serial, self-administered lateral flow devices as an alternative to self-isolation.medRxiv 2021:2021.03.23.21254168. [Preprint.] https://doi.org/10.1101/2021.03.23.21254168

45 Aggarwal DD, Fieldman DT. Genomic epidemiology of SARS-CoV-2 in the University of Cambridge identifies dynamics of transmission: an interim report. 2020. https://assets.publishing.service.gov.uk/government/uploads/system/uploads/attachment_data/file/950795/s0963-genomicepidemiology-sars-cov-2-university-of-cambridge.pdf

46 Cevik M, Baral SD, Crozier A, Cassell JA. Support for self-isolation is critical in covid-19 response. BMJ 2021;372:n224.pmid: 33504501

47 Kerkhoff AD, Sachdev D, Mizany S, etal. Evaluation of a novel community-based COVID-19 'Test-to-Care' model for low-income populations. PLoS One 2020;15:e0239400. doi: 10.1371/journal.pone.0239400. pmid: 33035216

48 Pettigrew LM, van Schalkwyk M, Rechel B, Garlick R. Where's the integration between public health and primary care in the response to covid-19? BMJ Opinion 18 Feb 2021. https://blogs.bmj.com/bmj/2021/02/18/wheres-the-integration-between-public-health-and-primary-care-in-the-response-to-covid-19/

49 Crozier A, Mckee M, Rajan S. Fixing England's COVID-19 response: learning from international experience. J R Soc Med 2020;113:422-7. doi: 10.1177/0141076820965533. pmid: 33058751

50 Harding-Edgar L, McCartney M, Pollock AM. Test and trace strategy has overlooked importance of clinical input, clinical oversight and integration. J R Soc Med 2020;113:428-32.pmid: 33108948

51 Wasserheit JN, Aral SO. The dynamic topology of sexually transmitted disease epidemics: implications for prevention strategies. J Infect Dis 1996;174(Suppl 2):S201-13. doi: 10.1093/infdis/174.Supplement_2.S201 pmid: 8843250

52 Mishra S, Baral SD. Rethinking the population attributable fraction for infectious diseases. Lancet Infect Dis 2020;20:155-7. doi: 10.1016/\$1473-3099(19)30618-8 pmid: 31753764

53 SPI-M-O.Consensus statement on covid-19. 12 May 2021. https://assets.publishing.service.gov.uk/government/uploads/system/uploads/attachment_data/file/986709/S1237_SPI-MO_Consensus_Statement.pdf

This article is made freely available for use in accordance with BMJ's website terms and conditions for the duration of the covid-19 pandemic or until otherwise determined by BMJ. You may use, download and print the article for any lawful, non-commercial purpose (including text and data mining) provided that all copyright notices and trade marks are retained. 